\title{
The Conventional Gait Model - Success and limitations
}

Author's name and affiliation

Richard Baker, Fabien Leboeuf, Julie Reay

University of Salford, UK.

Morgan Sangeux.

The Royal Children's Hospital, The Murdoch Childrens Research Institute, Melbourne, Australia.

\section{Abstract}

The Conventional Gait Model (CGM) is a generic name for a family of closely related and very widely used biomechanical models for gait analysis. After describing its history, the core attributes of the model are described followed by evaluation of its strengths and weaknesses. An analysis of the current and future requirements for practical biomechanical models for clinical and other gait analysis purposes which have been rigorously calibrated suggests that the CGM is better suited for this purpose than any other currently available model. Modifications are required, however, and a number are proposed.

\section{Keywords}

Clinical gait analysis, biomechanical modelling. 


\section{Introduction}

The Conventional Gait Model (CGM) is a generic name for a family of biomechanical models which emerged in the 1980s based on very similar principles and giving very similar results. It has a rather complex history (outlined below) and as a consequence has been referred to by a range of different names. The use of the name Conventional Gait Model is an attempt to emphasize the essential similarity of these models despite those different names.

For a number of reasons the CGM became the de facto standard for gait analysis in the 1990s, particularly in clinical and clinical research applications. Despite considerable strengths, technological advances have left aspects of the CGM looking quite outdated. The model, as originally formulated, also has a number of intrinsic limitations and, as these have become more widely appreciated, a variety of modifications and alternatives have been developed. Although the model can no longer be regarded as an industry wide standard as was once the case, many of the more established and respected clinical centres still prefer to use the model considering its strengths to outweigh its limitations.

After a brief summary of the historical development of the CGM this chapter will describe its characteristics and then assess its strengths and limitations, concluding with some suggestions as to how the model could be developed in future in order to address those limitations whilst preserving its strengths.

\section{History}

(Italicised words in this section are names that are sometimes used to refer to the CGM)

The origins of the model can be traced to the work of John Hagy in the laboratory established by David Sutherland (Sutherland and Hagy 1972) who digitised the positions of skin markings indicating anatomical landmarks from bi-planar movie stills. The coordinates were then used to compute a number of joint angles. Patrick Shoemaker extended this approach (Shoemaker 1978) to incorporate Ed Chao's ideas on representing three dimensional joint motion as Euler angles (Chao 1980). Jim Gage on a visit to San Diego prior to developing his own gait analysis laboratory at the Newington Hospital in Connecticut and a succession of engineers including Scott Tashman, Dennis Tyburski and Roy Davis (Davis et al. 1991) further developed the ideas in a number of ways. Perhaps the most important of these were the calculation of joint angles on the basis of estimated joint centres (rather than directly from marker locations) and the incorporation of three dimensional inverse dynamics to estimate joint moments (Ounpuu et al. 1991) based on the approach of David Winter (Winter and Robertson 1978). At about this time Murali Kadaba developed a very similar model at the Helen Hayes Hospital (Kadaba et al. 1990; Kadaba et al. 1989). There was communication between the two groups over this period but there are now different memories as to the extent of this collaboration and the precise role of the different individuals involved.

Although some minor modifications have been proposed since, the subsequent history is largely about how the model was distributed. The Helen Hayes Model was developed as a package and distributed across seven American hospitals. A little later Oxford Metrics (now Vicon), the manufacturers of Vicon movement analysis systems chose to develop their own version of the model (with support from individuals at both Newington and Helen Hayes). This was embedded within a package known as the Vicon Clinical Manager (VCM) and later developed as the Plug-in Gait (PiG) model for Workstation software. Most manufacturers of gait analysis systems produce some version of the model which go under a variety of names. Perhaps because of commercial sensitivities it is generally rather unclear what level of agreement there is between data processed with these alternative models. 
Perhaps the most important factor leading to the widespread adoption of the CGM was the prominence of Vicon measurement systems in clinical and academic gait analysis at this time with VCM and PiG being delivered alongside with their hardware. Many of the more established clinical services were founded at this time and most adopted VCM and continued to use PiG. Jim Gage became a strong advocate for clinical gait analysis and with Roy Davis and Silvia Ounpuu established extremely well regarded teaching courses first at Newington, then Gillette Children's Hospital which were based on what they regarded as the Newington Model. The model was also explained and validated in a number of key papers (Kadaba et al. 1990; Kadaba et al. 1989; Davis et al. 1991; Ounpuu et al. 1996; Ounpuu et al. 1991) in considerably more detail than any other model at the time. Thus by the early 2000s the CGM had become established as the predominant gait model for clinical and clinical research purposes and a large community of users had developed embodying a solid understanding of its strengths and limitations.

Since that time this status has diminished somewhat. A larger number of suppliers to the gait analysis market and the increasing ease of integrating different software has widened the options for data processing. There have been considerable and often justified criticism of the limitations of the CGM and a general failure of the CGM community to develop the model to address these issues. Despite this the model is still almost certainly the most widely used and understood single model within the clinical and clinical research community. 


\section{State of the art}

As stated above the CGM is actually a family of closely related models but for simplicity this section will be limited to a description of that embodied in the VCM and PiG which are identical and the most commonly used versions. It is arguable whether the CGM is a model at all as the word is now understood in biomechanics and it was originally described as "an algorithm for computing lower extremity joint motion" (Kadaba et al. 1990) and "a data collection and reduction technique" (Davis et al. 1991) when first described. In the sections below, however, a modern understanding of biomechanical modelling will be used to describe the underlying concepts.

\section{Model structure and anatomical segment definitions}

The model has seven segments linked in a chain by ball joints (three rotational degrees of freedom) in the sequence left foot, left tibia, left femur, pelvis, right femur, right tibia, right foot. An orthogonal coordinate system is associated with each segment. While the three segment axes are mathematically equivalent clinical convention is to define the segment alignment in terms of the alignment of a primary axis and the rotation about this as defined by some off-axis reference point. The primary axes for each segment is taken to be that linking the joints which attach it to the two neighbouring segments in the kinematic chain. Conceptually the segment axis systems are thus defined by specifying a primary axis and reference point for each. These are defined in Table 1.

\section{Marker placement to estimate anatomical segment position}

Markers are placed in such a way that the segment orientations can be estimated. When the model was developed optoelectronic measurements systems were limited to resolving a small number of markers and thus the minimum number of markers possible is used. This is based on the assumption that the proximal joint of any leg segment (all those other than the pelvis) is known from the position and orientation of the joint to which it is linked proximally. More distal segment orientations are dependent on the orientation of the more proximal segments and the model is thus often described as being hierarchical. Because of the difficulty in resolving more than two markers on the foot at the time when the model was developed it defined the orientation of its primary axis but not any rotation about this. The locations of markers are given in Table 2.

The hierarchical process requires a method for determining the location of the joints within each segment. The hip joint location within the pelvis coordinate system is specified by three equations (Davis et al. 1991) which are functions of leg length and ASIS to ASIS distance. These are measured during physical examination (although ASIS to ASIS distance can also be calculated from the marker positions during a static trial). The knee joint centre in the femur coordinate system is assumed to lie in the coronal plane at the point at which the lines from it to the hip joint centre and lateral femoral epicondyle are perpendicular and the distance between joint centre and epicondyle is half the measured knee width. The ankle joint centre within the tibia is specified analogously with respect to the lateral malleolus.

The wand markers (on both femur and tibia) are thus important to define the segmental coronal plane. Use of the wand (rather than a surface mounted marker) has two main purposes. The first is that wands (particularly those with a moveable ball and socket joint at the base) can be adjusted easily to define the correct plane. At least as important, however, is that by moving the marker away from the primary axis of the segment they make definition of the coronal plane much less sensitive to marker placement error or soft tissue artefact. Concerns have been expressed that the markers wobble but there is little evidence of this in gait data (it would appear as fluctuation in the hip rotation graph) if they are taped or strapped securely to the thigh. 
The foot segment uses the ankle joint centre (which has already been defined in the tibia coordinate system) and one forefoot or toe marker. The placement of this marker varies considerably with some centres placing quite distally (typically at the level of the metatarsophalangeal joint) in which case it indicates overall foot alignment. Other centres, particularly those dealing with clinical populations who often have foot deformities choose a more proximal placement (typically at the level of the cuneiforms) in order to give a better indication of hindfoot alignment. Placement of a heel marker during the static trial also allows for offsets to ensure that ankle measurements were aligned with the long axis of the foot rather than simply by the line from the ankle joint centre to the toe marker. A common variant is to calculate the plantarflexion offset on the assumption that the foot is flat, and thus that the long axis of the foot is in the horizontal plane, during the static trial.

\section{Kinematic outputs}

Kinematic outputs are mainly joint angles describing the orientation of the distal segment with respect to that of the proximal segment. The orientation of the pelvis is output as segment angles (with respect to the laboratory-based axis system) as is the transverse plane alignment of the foot (called foot progression). In three dimensions the orientation of one segment with respect to another must be represented by three numbers. The CGM uses Cardan angles which represent the set of sequential rotations about three different and mutually perpendicular axes that would rotate the distal segment from being aligned with the proximal segment (or the laboratory-based coordinate system) to its actual position.

In the original model the rotation sequence was about the medial-lateral, then the anteriorposterior and finally the proximal-distal axis for all joints (and segments). Although this sequence maps onto the conventional clinical understanding of the angles for most joints, it does not for the pelvis (Baker 2001). This is because with this rotation sequence, pelvic tilt is calculated as the rotation around the medial-lateral axis of the laboratory coordinate system, rather than the mediallateral axis of the pelvis segment, as per conventional understanding. Baker (Baker 2001) proposed to reverse the rotation sequence which results in pelvic angles that more closely map onto the conventional clinical understanding of these terms (confirmed by Foti et al. 2001). Following Baker's recommendation to use globographic angles (Baker 2011) these can be interpreted exactly as listed in Table 3.

Whilst not formally a part of the model, the CGM is closely associated with a particular format of gait graph (see Figure 1). All data is time normalised to one gait cycle and the left side data plotted in one colour (often red) and the right side data in another (often green, but blue reduces the risk of confusion by those who are colour blind). The time of toe off is denoted by a vertical line across the full height of the graph and opposite foot off and contact by tick marks at either the top or bottom of the graphs (in the appropriate colour). Normative data is often plotted as a grey band in the background (typical \pm one standard deviation about the mean). The graphs are then commonly displayed as arrays with the columns representing the different anatomical planes and the rows representing the different joints.

\section{Kinetic outputs}

The CGM is commonly used to calculate kinetic as well as kinematic outputs (Davis et al. 1991; Kadaba et al. 1989). Both the Newington and Helen Hayes approaches used inverse dynamics to estimate joint moments from force plate measurements of the ground reaction, an estimate of segment accelerations from kinematic data and estimates of segment inertial parameters. The main difference was that the Newington group took segment inertial parameters from the work of Dempster et al. (Dempster 1955) whereas the Helen Hayes group (Kadaba et al. 1989) took them 
from Hindrichs et al. (Hinrichs 1985) based on Clauser et al. (Clauser et al. 1969). Joint moments are fairly insensitive to these parameters (Rao et al. 2006; Pearsall and Costigan 1999) and it is unlikely that this would have led to noticeable differences in output. VCM and PiG used values from Dempster et al. (1955). Joint moments were presented in the segment coordinate systems. The early papers do not specify whether the proximal or distal segment was used for this. PiG and VCM allowed the user to select which (or to use the global coordinate system) and the default setting of the distal system is probably most widely used.

Joint power is also calculated as the vector dot product of the joint moment and angular velocity (note that this should be the true angular velocity vector and not that of the time derivatives of the Cardan angles). Power is a scalar quantity and there is thus no biomechanical justification for presenting "components" of power.

\section{Variants}

Over the years a number of variants to the CGM have been implemented by particular groups. Most of these have not been formally described in the academic literature.

- The original papers describing the model assumed that the femur and tibia wand markers could be placed accurately. Early experience was that this was challenging and an alternative technique was developed in which the markers were only positioned approximately and a Knee Alignment Device (KAD) was used during static trials to indicate the orientation of the knee flexion axis and hence the coronal plane of the femur. This allowed rotational offsets to be calculated to correct for any misalignment of the wand markers (with the tibial offset requiring an estimate of tibial torsion from the physical examination).

- A development within PiG allowed a medial malleolar marker to indicate the position of the trans-malleolar axis during the static trial and hence to calculate a value of tibial torsion rather than requiring this to be measured separately.

- A method of allowing for the thickness of soft tissues over the ASIS was provided by allowing the measurement of the ASIS to greater trochanter distance which is an estimate of the distance by which the hip joint centre was posterior to the base plate of the ASIS marker.

- A technique called DynaKAD has been proposed (Baker et al. 1999) to define the thigh rotation offset by minimising the varus-valgus movement during the walking trial. Other techniques have been used suggested to define this from functional calibration trials (Schwartz and Rozumalski 2005; Sauret et al. 2016; Passmore and Sangeux 2016).

- VCM and PiG introduced an angular offset along the tibia such that knee rotation is defined as being zero during a static trial when the KAD is used and the orientation of the ankle joint axis is defined by a measurement of tibial torsion made during the physical exam (rather than the tibial wand marker).

- Another development of PiG allowed the heel marker to be used to give an indication of inversion/eversion of the foot (rotation about the long axis) if it was left in place during the walking trial.

- A further development allowed an angular offset to be applied allowing for the foot being pitched forward by a known amount during a static trial (to take account of the pitch of a shoe for example).

- An upper body model was developed by Vicon which, though widely used, has never been rigorously validated. 


\section{Strengths}

Recent opinion has tended to emphasize the weaknesses of the CGM but it is also important to acknowledge its many strengths. In a world in which clinical governance is increasingly important the CGM has been more extensively validated than any other model in routine clinical use. The early papers of Kadaba et al. were considerably ahead of their time in their approach to validation. The basic description of the model (Kadaba et al. 1990) includes presentation of normative data, a comparison of this against normative data from a range of previous papers and a sensitivity analysis of the most common measurement artefact arising from the difficulty in placing thigh wands accurately. The follow up paper (Kadaba et al. 1989, which was actually published first!) is also a definitive repeatability study. 15 out of the 23 papers identified in the classic systematic review of repeatability studies of kinematic models of McGinley et al. (McGinley et al. 2009) used a variant of the CGM and a more recent study (Pinzone et al. 2014) has demonstrated the essential similarity of normative kinematic data collection from gait analysis services on different sides of the world but captured by the CGM. This body of formal validation literature is strongly reinforced by a large number of papers reporting use of the CGM in a very wide range of clinical and research applications. The CGM is thus particularly appropriate as a standardised and validated model for users who are more interested in interpreting what the results mean than in further model development and validation.

Although the implementation of the model is not trivial, the basic concepts are about as simple as possible for a clinically useful model. It uses a minimal markerset which can be applied efficiently in routine clinical practice. The model is deterministic (doesn't require any optimised fitting process) and thus the effects of marker misplacement and or soft tissue artefact are entirely predictable (Table 4 illustrates the effect that a given movement in each marker will affect outputs). It is thus possible to develop a comprehensive understanding of how the model behaves without being an expert in biomechanics. This can be logically extended to give clear indications of how marker placement can be best adapted in order to obtain clinically meaningful outputs in the presence of bone and joint deformities or devices such as orthoses and prostheses.

It is unfortunate, therefore, that in the early years the model developed a reputation for behaving as a "black box". This probably arose because the most commonly available implementation, in the VCM, incorporated some refinements to the previously published versions (e.g. the thigh and shank offsets) which were only described conceptually in the accompanying product documentation. Many people assumed that there was insufficient information to fully understand the model; an assumption proved false by a number of exact clones emerging (Baker et al. 1999 is an example).

\section{Weaknesses}

Accuracy. Whilst the CGM has been subjected to several studies to investigate its repeatability there have been very few studies of its accuracy and those have focussed on very specific issues such as the location of the hip joint centre location (Sangeux et al. 2014; Peters et al. 2012; Sangeux et al. 2011) and orientation of the knee flexion axis (Sauret et al. 2016; Passmore and Sangeux 2016) in standing. The model is intended to track the movements of the bones and there have been no studies performed to establish how accurately it can do this. This is principally because gold standard methods for tracking bone movement during walking are challenging (although a range of techniques are available - see section on Future Directions below). It should be emphasized, however, that this is a weakness of all commonly used biomechanical models for gait analysis and not just the CGM.

Hip joint centre position. A considerable body of knowledge now suggests that there are better methods for specifying the location of the hip joint centre within the pelvic coordinate system than 
those used within the CGM (Leardini et al. 1999; Sangeux et al. 2011; Sangeux et al. 2014; Harrington et al. 2007; Peters et al. 2012). Whilst the first of these (Leardini et al. 1999) suggested that functional calibration methods were superior to equations, more recent studies suggest that alternative equations can give results at least as good as functional methods in healthy adults (Sangeux et al. 2011; Sangeux et al. 2014; Harrington et al. 2007) and better in children with disabilities (Peters et al. 2012).

Defining the coronal plane of the femur. The first of the papers of Kadaba et al. (Kadaba et al. 1990) highlighted the sensitivity of the CGM to misplacement of the thigh markers leading to erroneous definition of the coronal plane of the femur. This leads to a well-known artefact in which the coronal plane knee kinematics show cross-talk from knee flexion-extension which is generally of little clinical significance but highlights uncertainty in hip rotation which is a major limitation of the model. Use of the KAD (which is very poorly documented in the literature) led to some improvements but this is still generally regarded as one of the most significant limitations of the model.

Over-simplistic foot modelling. Modelling the foot as single axis rather than three dimensional segment arose from the difficulty early models had in detecting more than one marker placed on a small foot. Whilst reliable detection of many markers on the foot has been possible for many years now, however, a formal extension of the model has never been proposed to model the foot more comprehensively. The Oxford Foot Model (Carson et al. 2001), which is probably now the most widely used in clinical and research practice, differs markedly from the CGM in that it allows translations between the forefoot, hindfoot and tibia (rather than the spherical joints that are a characteristic of the CGM).

Unconstrained segment dimensions. The CGM does not require the segments to be of a fixed length and soft-tissue artefact generally acts in such a way that the distance between the hip and knee joint centres can vary by as much as $2 \mathrm{~cm}$ over the gait cycle during walking. Whilst this probably has a small effect on kinematic and kinetic outputs it does prevent the use of the model with more advanced modelling techniques such as muscle length modelling and forward dynamics for which a rigid linked segment model is required. Modern inverse kinematic techniques (Lu and O'Connor 1999) which depend on rigid linked segment models also offer the potential to incorporate modelling of soft-tissue artefact (Leardini et al. 2005) based on data such as fluoroscopy studies (Tsai et al. 2009; Akbarshahi et al. 2010) in a manner that is not possible within the CGM.

Inadequate compensation for soft tissues over pelvic landmarks. Whilst methods have been proposed for measuring and taking into account the soft tissues over pelvic landmarks, none are particularly convincing or validated. As populations, particularly those with limited walking abilities, become increasingly overweight this becomes a more important problem.

Poorly validated upper body model. Whilst Davis et al. (Davis et al. 1991) did suggest placement of markers on the shoulders to give an indication of trunk alignment this has not been widely implemented. Vicon developed an upper body model for PiG but, despite this being quite widely used, there have been no published validations of its outputs. It is still not clear how important upper limb movements are in relation to clinical gait analysis but a knowledge of trunk alignment and dynamics is clearly important to understand the mechanics of the gait patterns of many people with a range of conditions.

\section{Alternatives}

Perhaps the most commonly used alternatives to the CGM are 6 degree of freedom (6DoF) models. These can be traced back to the work of Cappozzo et al. (Cappozzo et al. 1995) and have been 
popularised through Visual3D software (C-motion, Kingston, Canada). They track the segments independently (without constraining the joints) and can be based on skin mounted markers (as implied by the illustration in the original paper) or rigid marker clusters (as is more common nowadays). Perhaps the most important limitation of this approach is that it refers to a modelling technique rather than any specific model (CAST is an abbreviation for calibrated anatomical landmark technique) and no specific model has been widely used and rigorously validated. The Cleveland Clinic Marker Set was an early example which achieved popularity when it was implemented in the Orthotrack Software (Motion Analysis Corporation, Santa Rosa, USA) but has never been validated (or even fully described) in the peer-review literature. More recently Leardini et al. (Leardini et al. 2007) published and validated the IOR model but there are only limited reports of use outside Bologna in the literature (and it is worth noting that the IOR model, in using skin mounted markers, differs quite markedly from most contemporary 6DoF modelling which uses rigid clusters).

6DoF models are sometimes presented as addressing the known limitations of the CGM. Sometimes there is justification in these claims (e.g. the segments are fixed length) but often corresponding issues are overlooked (e.g. non-physiological translations between the proximal and distal bones at some joints). Soft tissue artefact between markers is certainly eliminated by using rigid clusters but a different form of soft tissue artefact will affect the orientation and position of the whole cluster in relation to the bones (Barre et al. 2013). Other issues such as the difficulty in estimating the hip joint centre or knee axis alignment affects all models. One advantage of most 6DoF models is that they use medial and lateral epicondyle markers during a static trial to define the knee joint axis. This may be more repeatable than precise alignment of thigh wands or KADs. It is also worth noting that this is only a difference of knee calibration technique which could easily be incorporated into the CGM.

Inverse kinematic (often referred to as kinematic fitting or global optimisation) models have also been reported (Lu and O'Connor 1999; Reinbolt et al. 2007; Charlton et al. 2004) and this approach has become more popular since it was incorporated within OpenSim (Seth et al. 2011) as the default technique for tracking marker data. In this, a linked segment rigid body model is defined and an optimisation technique is used to fit the model to the measured marker positions, generally using some weighted least-squares fit cost function. As with 6 DoF models this approach has advantages and disadvantages with respect to the CGM. It is also similar to the 6DoF approach in that no single model has received widespread use or been subject to rigorous validation. The approach is inherently compatible with advanced modelling techniques (e.g. muscle length modelling and forward dynamics) and is well suited to either stochastic or predictive approaches to modelling soft tissue artefact. It's most notable weakness is that it is non-deterministic and on occasions artefacts can arise in the data from soft-tissue artefact, marker misplacement or erroneous model definition that can be extremely difficult to source. On balance, however, it is likely that future developments will be based on an inverse kinematic approach.

\section{Future Directions}

Over the lifetime of the CGM the nature of gait analysis has changed considerably in at least two important ways. The first is the growing importance of clinical governance (Scally and Donaldson 1998) and evidence based practice within healthcare organisations. This requires increasing standardisation of all operations based upon well validated procedures. The emergence of accreditation schemes such as those now operated by the Clinical Movement Analysis Society (CMAS, UK and Ireland) or the Committee for Motion Laboratory Accreditation (USA) are a consequence of this. At present the focus is on whether written protocols exist at all but it is inevitable, as this minimal standard becomes universally implemented, that more attention will be 
paid to ensuring that any procedures are appropriately validated. This may be reinforced by more rigorous implementation of medical device legislation to gait analysis software which should require manufacturers to ensure that clinically relevant outputs (such as joint angles from a specific biomechanical model) are reproducible (rather than just the technical outputs such as marker locations).

The other change, which has implications beyond gait analysis for purely clinical purposes, is that gait analysis systems are getting much cheaper and more user friendly. It can no longer be assumed that laboratories will have a staff member suitably qualified in biomechanics to create and adapt their own models. People using current technology generally want to implement standardised techniques allowing them to focus on the interpretation of data rather than on developing individualised solutions and being distracted by the challenge of their validation. Such users will require a model that is simple enough to be understood conceptually in sufficient detail to guide quality assurance and interpretation of the data produced. In scientific research it would also be useful to have a widely accepted standardised approach to capturing data to ensure that results from different centres are as comparable as possible.

For clinical users and those in other fields who want to focus on the interpretation of data rather than the mechanics of data capture therefore, there is a real need for a widely accepted, standardised and validated approach to data capture (including biomechanical modelling) which is efficient and robust in application and sufficiently simple to be understood by the users themselves (rather than relying on biomechanical experts). To be useful in this context it needs to be widely applicable to all people who are old enough to walk and who have a range of different health conditions (or none). There needs to be a strong evidence base for the reproducibility of measurements, specific training for staff involved in the capture and processing of data and appropriate metrics to assure the quality of measurements in routine practice.

The CGM satisfies all of these requirements at least as well, and in most cases considerably better, than alternatives. Despite this, many users are frustrated by its limitations whilst potential users are often put off by its commonly perceived weaknesses (some justified, some not). It is clear that if the CGM is to have a future it will require modifications to address these.

A particular issue for the CGM is that many older laboratories have databases stretching back over considerable periods of time (several decades in many cases) and backward compatibility is perceived as extremely important. Ensuring rigorous backwards is incompatible with improving the modelling of course so a compromise is required. The most obvious is to ensure that any new model uses the same anatomical segment definitions (see Table 1) as the original. It may be that modifications lead to systematic differences with the original CGM, but it will be clear that these are a consequences of improvements in the modelling rather than redefinition of what is being measured. It will also be important to quantify any such systematic changes in order that they can be accounted for if data processed using different versions of the model can be compared.

Another specific issue with the CGM is the perception of it as a "black box" processing technique which cannot be properly understood. This has persisted despite increasingly good documentation being produced but will be best addressed by publishing the actual computer code through which the model is implemented. Implementing the code in an open source language (such as Python) which is available to all users will also be important. Training and education packages will also be required for those less technically minded.

The specific modifications that are indicated would be: 
- Adoption of a robust inverse kinematic fitting approach based around a linked rigid segment model that is compatible with advanced musculoskeletal modelling techniques.

- Replacement of wand markers with a limited number of skin mounted tracking markers on the femur and tibia positioned to minimise sensitivity to soft tissue artefact (Peters et al. 2009) or marker misplacement.

- Incorporation of more accurate equations for estimating the hip joint centre and techniques for accounting for the depth of soft tissues anatomical landmarks on the pelvis.

- Improved methods for determining the orientation of the coronal plane of the femur. Basing this upon the position of medial and lateral femoral epicondyle markers during a calibration trial may be an improvement and functional calibration of the knee should be implemented as a quality assurance measure.

- Improvement of foot modelling by formalising the PiG approach to using the heel marker to give an indication of inversion and eversion of about the long axis of the foot. There is a lack of standardisation in where the forefoot (toe) marker is placed. Opting for a more proximal placement (at about the level of the tarsometatarsal joints) would lead to the foot representing movement of the hindfoot and open the possibility for some indication of forefoot alignment in relation to this using markers placed on the metarsophalangeal joints.

- Validation of an appropriate trunk model should be regarded as essential. Doing so on the basis of force plate measurements of centre of mass displacement during walking (Eames et al. 1999) would be useful to establish just how important measuring upper limb movement is in gait analysis.

Future versions should be adequately validated in line with a modern understanding of clinical best practice. At a minimum this should include evidence of reproducibility of results but it would also be useful to have accuracy established with reference to a variety of static and dynamic imaging techniques such as 3-d ultrasound (Peters et al. 2010; Hicks and Richards 2005; Passmore and Sangeux 2016), low intensity biplanar x-rays (Pillet et al. 2014; Sangeux et al. 2014; Sauret et al. 2016) or fluoroscopy (Tsai et al. 2009; Akbarshahi et al. 2010). There should also be publication of benchmark data with which services can compare their own to ensure consistency (Pinzone et al. 2014) and streamlined processed for conducting in-house repeatability studies would also be extremely useful. 


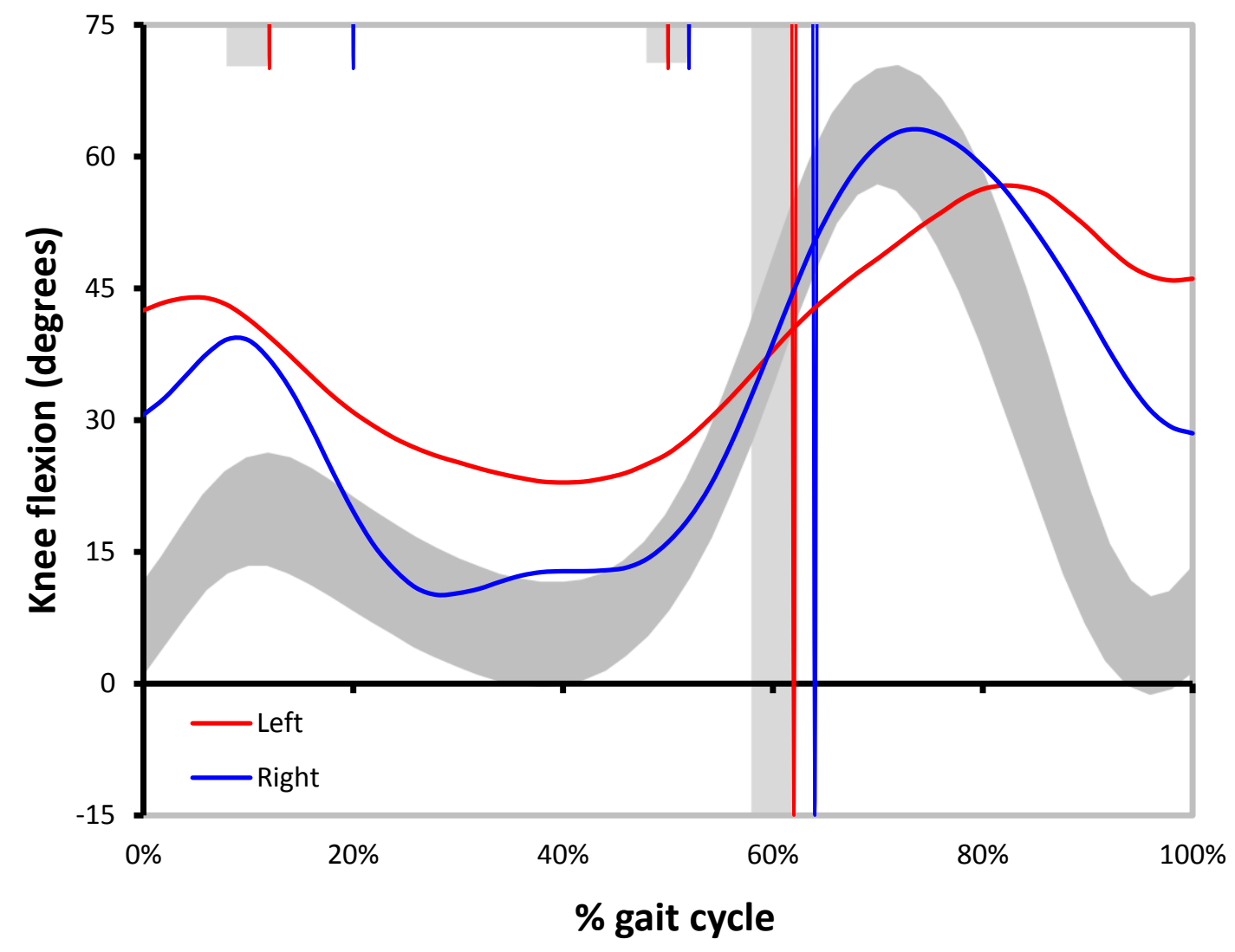

Figure 1. A standard gait graph. The curves represent how a single gait variable varies over the gait cycle. The vertical lines across the full height of the graph represents foot-off and the tick marks represent opposite foot off (to the left of graph) and opposite foot contact (to the right). Line in red are for the left side and in blue are for the left side. The grey areas represent the range of variability in some reference population as 1 standard deviation either side of the mean value. 


\begin{tabular}{|c|c|}
\hline & $\begin{array}{l}\text { Pelvis } \\
\text { The primary axis is the mediolateral axis running } \\
\text { from one hip joint centre to the other. In most } \\
\text { clinical applications it is assumed that the pelvis is } \\
\text { symmetrical and that this axis is thus parallel to the } \\
\text { line running from one anterior superior iliac spine } \\
\text { (ASIS) to the other. } \\
\text { The reference point for rotation about this axis is the } \\
\text { mid-point of the posterior superior iliac spines (PSIS). }\end{array}$ \\
\hline & $\begin{array}{l}\text { Femur } \\
\text { The primary axis is that running from the hip joint } \\
\text { centre to knee joint centre. } \\
\text { The reference point is the lateral epicondyle. } \\
\text { For validation purposes: } \\
\text { - the hip joint centre will be taken as the } \\
\text { geometrical centre of a sphere fitted to the } \\
\text { articular surface of the femoral head. } \\
\text { the knee joint centre will be taken as the mid- } \\
\text { point of the medial and lateral epicondyles. } \\
\text { These are often difficult to palpate, however, } \\
\text { and for some purposes the line between these } \\
\text { landmarks will be assumed to be parallel to } \\
\text { that linking the most posterior aspects of the } \\
\text { femoral condyles. }\end{array}$ \\
\hline & $\begin{array}{l}\text { Tibia } \\
\text { The primary axis is that running from the knee joint } \\
\text { centre to ankle joint centre. } \\
\text { The reference point is the lateral malleolus. } \\
\text { For validation purposes: } \\
\text { - the ankle joint centre will be assumed to be the } \\
\text { mid-point of the medial and lateral } \\
\text { epicondyles. }\end{array}$ \\
\hline$\frac{\sum^{2} x^{2}}{x^{2}}$ & $\begin{array}{l}\text { Foot } \\
\text { The primary axis is that running from the most } \\
\text { posterior axis of the calcaneus along the second ray } \\
\text { and parallel to the plantar surface of the foot. } \\
\text { Rotation about this axis is not defined. }\end{array}$ \\
\hline
\end{tabular}




\begin{tabular}{|c|c|}
\hline & $\begin{array}{l}\text { Pelvis } \\
\text { Markers are placed over both ASIS and PSIS in order } \\
\text { that they lie in the plane containing the anatomical } \\
\text { landmarks. } \\
\text { A set of equations are used to estimate the location } \\
\text { of the hip joint within the pelvic coordinate system. }\end{array}$ \\
\hline & $\begin{array}{l}\text { Femur } \\
\text { The hip joint centre within the femur is coincident } \\
\text { with that within the pelvis. } \\
\text { A marker is placed over the lateral femoral } \\
\text { epicondyle and another on a wand on the lateral } \\
\text { thigh in such a way that the two markers and the hip } \\
\text { joint centre lie within the coronal plane of the femur. } \\
\text { The knee joint centre is to be defined such that it, } \\
\text { the hip joint centre and the epicondyle marker form } \\
\text { a right angle triangle within the coronal plane of the } \\
\text { femur with a base of half the measured knee width. }\end{array}$ \\
\hline & $\begin{array}{l}\text { Tibia } \\
\text { The knee joint centre within the tibia is coincident } \\
\text { with that within the femur. } \\
\text { A marker is placed over the lateral malleolus and } \\
\text { another on a wand on the lateral leg in such a way } \\
\text { that the two markers and the knee joint centre lie } \\
\text { within the coronal plane of the tibia. } \\
\text { The ankle joint centre is to be defined such that it, } \\
\text { the knee joint centre and the malleolar marker form } \\
\text { a right angle triangle within the coronal plane of the } \\
\text { tibia with a base of half the measured ankle width. }\end{array}$ \\
\hline $\operatorname{lag}$ & $\begin{array}{l}\text { Foot } \\
\text { The ankle joint centre in the foot is defined to be } \\
\text { coincident with that with the tibia. } \\
\text { A marker is placed on the forefoot. } \\
\text { Another is placed on the posterior aspect of the heel } \\
\text { for the static trial such that the line between the two } \\
\text { makers is parallel to the long axis of the foot. The } \\
\text { angles between this and the line from the ankle joint } \\
\text { centre to the forefoot marker in the sagittal and } \\
\text { horizontal planes are calculated. } \\
\text { The heel marker is not used in walking trials but the } \\
\text { offsets are used to estimate the alignment of the } \\
\text { long axis of the foot based on the line between ankle } \\
\text { joint centre and forefoot marker. }\end{array}$ \\
\hline
\end{tabular}


Pelvis (with respect to global coordinate system)

Internal/external rotation: rotation of the medio-lateral axis about the vertical axis

Obliquity (up/down):

rotation of the medio-lateral axis out of the horizontal plane

Anterior/posterior tilt:

rotation around the medio-lateral axis

Hip (femur with respect to pelvis coordinate system)

Flexion/extension:

rotation of the proximal distal axis about the medio-lateral axis

Ad/abduction:

Internal/external rotation:

rotation of the proximal-distal axis out of the sagittal plane

rotation around the proximal-distal axis

Knee (tibia with respect to femur coordinate system)

Flexion/extension:

rotation of the proximal distal axis about the medio-lateral axis

Ad/abduction:

Internal/external rotation:

rotation of the proximal-distal axis out of the sagittal plane rotation around the proximal-distal axis

Ankle (foot with respect to tibia coordinate system)

Dorsiflexion/plantarflexion: rotation of the proximal distal axis about the medio-lateral axis Internal/external rotation: rotation of the proximal-distal axis out of the sagittal plane

Foot (with respect to global coordinate system)

Foot progression (in/out):

rotation of the proximal-distal axis out of the "sagittal" plane 
Table 4 Effects of moving a marker $5 \mathrm{~mm}$ in the specified direction on the outputs of the CGM. Note that because of the hierarchical basis of the model that movements can only affect segments on or below that to which a given marker is attached. Changes in angle of less than $0.1^{\circ}$ are left blank.

\begin{tabular}{|c|c|c|c|c|c|c|c|c|c|c|c|c|}
\hline \multirow{2}{*}{$\begin{array}{l}\text { Marker } \\
\text { moved }\end{array}$} & \multicolumn{3}{|c|}{ Pelvis } & \multicolumn{3}{|c|}{ Hip } & \multicolumn{3}{|c|}{ Knee } & \multicolumn{2}{|c|}{ Ankle } & \multirow{2}{*}{$\begin{array}{c}\text { Foot } \\
\text { Internal } \\
\text { progression }\end{array}$} \\
\hline & Tilt & Obliquity & Rotation & Flexion & Adduction & $\begin{array}{l}\text { Internal } \\
\text { rotation }\end{array}$ & Flexion & Varus & $\begin{array}{l}\text { Internal } \\
\text { rotation }\end{array}$ & Dorsiflexion & $\begin{array}{l}\text { Internal } \\
\text { rotation }\end{array}$ & \\
\hline RASI up & -0.9 & 1.4 & 0.1 & -1.2 & 1.8 & -0.5 & -0.2 & -0.4 & & & & \\
\hline RASI out & 0.4 & & 0.2 & -0.1 & & -0.3 & -0.2 & -0.1 & & & & \\
\hline SACR up & 1.8 & & & 2 & & & 0.2 & 0.1 & -0.3 & & -0.1 & -0.1 \\
\hline \multicolumn{13}{|l|}{ SACR out } \\
\hline \multicolumn{13}{|l|}{ RTHI up } \\
\hline RKAD int & & & & -0.5 & -0.1 & 2.8 & -0.9 & 1 & -0.1 & -0.1 & -2.7 & \\
\hline RKNE up & & & & & & & -0.2 & -0.1 & 0.4 & -0.2 & -0.4 & 0 \\
\hline RKNE ant & & & & 1.3 & 0.1 & -1.8 & 2.2 & -0.9 & & 0.8 & 1.9 & 0.1 \\
\hline \multicolumn{13}{|l|}{ RTIB up ${ }^{1}$} \\
\hline \multicolumn{13}{|l|}{ RTIB ant ${ }^{1}$} \\
\hline RANK up & & & & & & & & & & 0.1 & 0.1 & 0.1 \\
\hline RANK ant & & & & & & & -0.9 & & -0.1 & -1 & -1.1 & 0.1 \\
\hline RTOE out & & & & & & & & & & 0.1 & -4.6 & 4.7 \\
\hline RTOE ant & & & & & & & & & & & & \\
\hline
\end{tabular}




\section{References}

Akbarshahi M, Schache AG, Fernandez JW, Baker R, Banks S, Pandy MG (2010) Non-invasive assessment of soft-tissue artifact and its effect on knee joint kinematics during functional activity. J Biomech 43 (7):1292-1301. doi:10.1016/j.jbiomech.2010.01.002

Baker R (2001) Pelvic angles: a mathematically rigorous definition which is consistent with a conventional clinical understanding of the terms. Gait Posture 13 (1):1-6. doi:10.1016/S0966-6362(00)00083-7

Baker R (2011) Globographic visualisation of three dimensional joint angles. J Biomech 44 (10):18851891. doi:10.1016/j.jbiomech.2011.04.031

Baker R, Finney L, Orr J (1999) A new approach to determine the hip rotations profile from clinical gait analysis data. Hum Mov Sci 18:655-667. doi:DOI: 10.1016/S0167-9457(99)00027-5

Barre A, Thiran JP, Jolles BM, Theumann N, Aminian K (2013) Soft tissue artifact assessment during treadmill walking in subjects with total knee arthroplasty. IEEE Trans Biomed Eng 60 (11):3131-3140. doi:10.1109/TBME.2013.2268938

Cappozzo A, Catani F, Croce UD, Leardini A (1995) Position and orientation in space of bones during movement: anatomical frame definition and determination. Clin Biomech (Bristol, Avon) 10 (4):171-178. doi:10.1016/0268-0033(95)91394-T

Carson MC, Harrington ME, Thompson N, O'Connor JJ, Theologis TN (2001) Kinematic analysis of a multi-segment foot model for research and clinical applications: a repeatability analysis. J Biomech 34 (10):1299-1307. doi:10.1016/S0021-9290(01)00101-4

Chao EY (1980) Justification of triaxial goniometer for the measurement of joint rotation. J Biomech 13:989-1006. doi:10.1016/0021-9290(80)90044-5

Charlton IW, Tate P, Smyth P, Roren L (2004) Repeatability of an optimised lower body model. Gait Posture 20 (2):213-221. doi:10.1016/j.gaitpost.2003.09.004

Clauser C, McConville J, Young J (1969) Weight volume and centre of mass of segments of the human body (AMRL Technical Report). Wright-Patterson Air Force Base, $\mathrm{OH}$,

Davis RB, Ounpuu S, Tyburski D, Gage J (1991) A gait analysis data collection and reduction technique. Hum Mov Sci 10:575-587. doi:10.1016/0167-9457(91)90046-Z

Dempster W (1955) Space requirements of the seated operator (WADC Technical Report :55-159). Wright-Patterson Airforce Base, $\mathrm{OH}$.,

Eames M, Cosgrove A, Baker R (1999) Comparing methods of estimating the total body centre of mass in three-dimensions in normal and pathological gait. Hum Mov Sci 18:637-646. doi:10.1016/S0167-9457(99)00022-6

Foti T, Davis RB, Davids JR, Farrell ME (2001) Assessment of methods to describe the angular position of the pelvis during gait in children with hemiplegic cerebral palsy. Gait Posture 13:270

Harrington ME, Zavatsky AB, Lawson SE, Yuan Z, Theologis TN (2007) Prediction of the hip joint centre in adults, children, and patients with cerebral palsy based on magnetic resonance imaging. J Biomech 40 (3):595-602. doi:10.1016/j.jbiomech.2006.02.003

Hicks JL, Richards JG (2005) Clinical applicability of using spherical fitting to find hip joint centers. Gait Posture 22 (2):138-145. doi:10.1016/j.gaitpost.2004.08.004

Hinrichs RN (1985) Regression equations to predict segmental moments of inertia from anthropometric measurements: an extension of the data of Chandler et al. (1975). J Biomech 18 (8):621-624. doi:10.1016/0021-9290(85)90016-8

Kadaba MP, Ramakrishnan HK, Wootten ME (1990) Measurement of lower extremity kinematics during level walking. J Orthop Res 8 (3):383-392. doi:10.1002/jor.1100080310

Kadaba MP, Ramakrishnan HK, Wootten ME, Gainey J, Gorton G, Cochran GV (1989) Repeatability of kinematic, kinetic, and electromyographic data in normal adult gait. J Orthop Res 7 (6):849860. doi:10.1002/jor.1100070611 
Leardini A, Cappozzo A, Catani F, Toksvig-Larsen S, Petitto A, Sforza V, Cassanelli G, Giannini S (1999) Validation of a functional method for the estimation of hip joint centre location. J Biomech 32 (1):99-103. doi:10.1016/S0021-9290(98)00148-1

Leardini A, Chiari L, Della Croce U, Cappozzo A (2005) Human movement analysis using stereophotogrammetry. Part 3. Soft tissue artifact assessment and compensation. Gait Posture 21 (2):212-225. doi:10.1016/j.gaitpost.2004.05.002

Leardini A, Sawacha Z, Paolini G, Ingrosso S, Nativo R, Benedetti MG (2007) A new anatomically based protocol for gait analysis in children. Gait Posture 26 (4):560-571. doi:10.1016/j.gaitpost.2006.12.018

Lu TW, O'Connor JJ (1999) Bone position estimation from skin marker co-ordinates using global optimisation with joint constraints. J Biomech 32 (2):129-134. doi:10.1016/\$00219290(98)00158-4

McGinley JL, Baker R, Wolfe R, Morris ME (2009) The reliability of three-dimensional kinematic gait measurements: a systematic review. Gait Posture 29 (3):360-369. doi:10.1016/j.gaitpost.2008.09.003

Ounpuu O, Davis R, Deluca P (1996) Joint kinetics: Methods, interpretation and treatment decisionmaking in children with cerebral palsy and myelomeningocele. Gait Posture 4:62-78. doi:10.1016/0966-6362(95)01044-0

Ounpuu S, Gage J, Davis R (1991) Three-dimensional lower extremity joint kinetics in normal pediatric gait. J Pediatr Orthop 11:341-349

Passmore E, Sangeux M (2016) Defining the medial-lateral axis of an anatomical femur coordinate system using freehand 3D ultrasound imaging. Gait Posture 45:211-216. doi:10.1016/j.gaitpost.2016.02.006

Pearsall DJ, Costigan PA (1999) The effect of segment parameter error on gait analysis results. Gait Posture 9 (3):173-183. doi:The effect of segment parameter error on gait analysis results

Peters A, Baker R, Morris ME, Sangeux M (2012) A comparison of hip joint centre localisation techniques with 3-DUS for clinical gait analysis in children with cerebral palsy. Gait Posture 36 (2):282-286. doi:10.1016/j.gaitpost.2012.03.011

Peters A, Baker R, Sangeux M (2010) Validation of 3-D freehand ultrasound for the determination of the hip joint centre. Gait Posture 31:530-532. doi:10.1016/j.gaitpost.2010.01.014

Peters A, Sangeux M, Morris ME, Baker R (2009) Determination of the optimal locations of surfacemounted markers on the tibial segment. Gait Posture 29 (1):42-48. doi:10.1016/j.gaitpost.2008.06.007

Pillet H, Sangeux M, Hausselle J, El Rachkidi R, Skalli W (2014) A reference method for the evaluation of femoral head joint center location technique based on external markers. Gait Posture 39 (1):655-658. doi:10.1016/j.gaitpost.2013.08.020

Pinzone O, Schwartz MH, Thomason P, Baker R (2014) The comparison of normative reference data from different gait analysis services. Gait Posture 40 (2):286-290. doi:10.1016/j.gaitpost.2014.03.185

Rao G, Amarantini D, Berton E, Favier D (2006) Influence of body segments' parameters estimation models on inverse dynamics solutions during gait. J Biomech 39 (8):1531-1536. doi:10.1016/j.jbiomech.2005.04.014

Reinbolt JA, Haftka RT, Chmielewski TL, Fregly BJ (2007) Are patient-specific joint and inertial parameters necessary for accurate inverse dynamics analyses of gait? IEEE Trans Biomed Eng 54 (5):782-793. doi:10.1109/TBME.2006.889187

Sangeux M, Peters A, Baker R (2011) Hip joint centre localization: Evaluation on normal subjects in the context of gait analysis. Gait Posture 34 (3):324-328. doi:10.1016/j.gaitpost.2011.05.019

Sangeux M, Pillet H, Skalli W (2014) Which method of hip joint centre localisation should be used in gait analysis? Gait Posture 40 (1):20-25. doi:10.1016/j.gaitpost.2014.01.024

Sauret C, Pillet H, Skalli W, Sangeux M (2016) On the use of knee functional calibration to determine the medio-lateral axis of the femur in gait analysis: Comparison with EOS biplanar radiographs as reference. Gait Posture 50:180-184. doi:10.1016/j.gaitpost.2016.09.008 
Scally G, Donaldson L (1998) Clinical governance and the drive for quality improvement in the new NHS in England. Br Med J 317:61-65. doi:10.1136/bmj.317.7150.61

Schwartz MH, Rozumalski A (2005) A new method for estimating joint parameters from motion data. J Biomech 38 (1):107-116. doi:10.1016/j.jbiomech.2004.03.009

Seth A, Sherman M, Reinbolt JA, Delp SL (2011) OpenSim: a musculoskeletal modeling and simulation framework for in silico investigations and exchange. Procedia IUTAM 2 (0):212232. doi:10.1016/j.piutam.2011.04.021

Shoemaker P (1978) Measurements of Relative Lower Body Segment Positions in Gait Analysis. MSc, University of California, San Diego

Sutherland D, Hagy J (1972) Measurement of gait movements from motion picture film. Journal of Bone and Joint Surgery 54A (4):787-797

Tsai T-Y, Lu T-W, Kuo M-Y, Hsu H-C (2009) Quantification of three-dimensional movement of skin markers realtive to the underlying bones during functional activities. Biomedical Engineering: Applications, Basis and Communications 21 (3):223-232. doi:10.4015/S1016237209001283

Winter D, Robertson D (1978) Joint torque and energy patterns in normal gait. Biol Cybern 29:137142. doi:10.1007/BF00337349 